can occur in some patients with warm AIHA, which can be differentiated from cold autoagglutination that occurs in cold AIHA by the reversal of agglutination by warming to $37^{\circ} \mathrm{C}$ in the latter, but not in the warm type [5].

Investigations showed that the patient had severe haemolytic anaemia on presentation, the haemoglobin was $6.2 \mathrm{~g} / \mathrm{dl}$, with a positive haemolytic screen of reticulocytosis, indirect hyperbilirubinaemia and erythroid hyperplasia in the bone marrow. According to the blood picture it is a spherocytic anaemia and the positive DAT indicates that it is immune mediated. Since it is positive for both C3d and IgG, it is considered as warm autoimmune hemolytic anaemia. However, subsequently her urine was strongly positive for haemosiderin which showed evidence of intravascular haemolysis.

\section{References}

1. Crowther M, Chan YL, Garbett IK, et al. Evidence based focused review of the treatment of Idiopathic Warm haemolytic anaemia in adults. Blood 2011; 118: 4036-40.

2. Michel M, Classification and therapeutic approaches in autoimmune haemolytic anaemia: an update. Expert Review of Hematology 2011; 4: 607-18.

3. Hoffbrand AV, Catovsky D, Tudenham EGD. Postgratuate Haematology; 6th Edition Wiley- Blackwell Ltd.

4. Packman $\mathrm{CH}$, Haemolytic anaemia due to warm autoantibodies. Blood Reviews 2008; 22: 17-31.

5. Petz LD. Cold antibody autoimmune haemolytic anaemias. Blood Reviews 2008; 22: 1-15.

\title{
A patient with severe body aches without fever progressing to dengue shock syndrome
}

\author{
R Premaratna $^{1}$, J K N D Miththinda ${ }^{2}$, C Abeyrathna ${ }^{2}$, H J de Silva ${ }^{1}$
}

Ceylon Medical Journal 2013; 58: 178-9

\section{Introduction}

More than $80 \%$ of dengue infections are asymptomatic, only 5\% develop more severe illness and an even smaller proportion develops life-threatening complications $[1,2]$. Characteristically dengue presents as an acute febrile illness associated with headache, myalgia, arthralgia and a rash, and typically goes through three phases; febrile, critical and recovery [2]. Several atypical manifestations of dengue such as neurological, cardiac, gastrointestinal, respiratory and renal have been reported [3].

We report a case of dengue shock syndrome (DSS), presenting with myalgia and arthralgia, but no history of fever.

\section{Case report}

A 23-year old previously healthy male with three days of body aches, headache, lethargy and nausea, but no documented fever was admitted to hospital due to insistence of the patient and relatives. There was no abdominal pain, vomiting or bleeding manifestations. He had self-medicated with paracetamol $1 \mathrm{~g}$ on an irregular basis for body aches. He had not consumed NSAIDs or steroids.

He was dark in complexion. He was afebrile, pulse rate was 98 beats /minute regular and the blood pressure (BP) was 110/70 mmHg in the sitting position. There was 4 $\mathrm{cm}$ tender hepatomegaly but no splenomegaly or free fluid in the abdomen. The chest and the central nervous system examination were normal.

The following day (day five of the illness) he developed postural dizziness and became drowsy, the BP was 90/60 $\mathrm{mmHg}$ and pulse rate was100/minute. Resuscitation was carried out with IV crystalloid boluses. He remained afebrile but the body aches persisted. On day six of the illness he developed sub conjunctival haemorrhages, gum bleeding and a right sided pleural effusion. The platelet count dropped to $3 \times 10^{9} / \mathrm{l}$, $\mathrm{Hb}$ rose $16 \%$ and PCV $13 \%$ and the AST rose to $251 \mathrm{iu} / \mathrm{l}$. At this point he developed the only documented fever spike of $101^{\circ} \mathrm{F}$. Dengue Ig M antibodies were positive.

Departments of ${ }^{1}$ Medicine and ${ }^{2}$ Public Health, Faculty of Medicine, University of Kelaniya, Sri Lanka.

Correspondence: RP, e-mail: <ranjanp64@gmail.com>. Received 8 January 2013 and revised version accepted 16 June 2013. Competing interests: none declared. 
From day seven onwards the clinical condition progressively improved with rise in platelets, WBC, reduction in PCV, Hb and liver enzymes. By day nine of illness, he was stable enough to be discharged.

\section{Discussion}

This patient had severe body aches and joint pain as the only presenting feature of dengue. The only documented fever spike was observed when he developed a blood pressure drop, third space fluid accumulation, rise in $\mathrm{Hb}, \mathrm{PCV}$, lowering of platelet counts and minor bleeding diathesis and rise in hepatic enzymes, all suggesting early dengue shock syndrome (DSS) and dengue haemorrhage fever (DHF III). At this point he was resuscitated with crystalloids and colloids according to WHO guidelines [2].

Although most dengue patients are asymptomatic, there are no documented reports of serious manifestations in afebrile patients. Our patient had no fever throughout the illness, except on the day of fluid leakage. In dengue, body aches and pains can be very severe but almost all patients with severe body aches develop fever during the initial phase of the illness and fever lasts 5-7 days in most patients. In this patient, severe body aches and pains were the main presenting symptoms. The CPK levels were normal and ESR was normal, and he therefore had no evidence of myositis. Admission to hospital would have prevented development of serious complications as the patient had to be resuscitated with fluids immediately upon developing features of DSS. We feel that clinicians should be aware of the possibility of severe dengue in patients living in endemic areas presenting with severe body aches in the absence of fever.

\section{References}

1. Whitehorn J, Farrar J. Dengue. British Medical Bulletin 2010; 95: 161-73.

2. World Health Organisation. Comprehensive guidelines for prevention and control of dengue and dengue haemorrhagic fever. Revised and expanded edition. World Health Organisation 2011.

3. Gulati S, Maheshwari A. Atypical manifestations of Dengue. Tropical Medicine and International Health 2007; 12: 1087-95. 